\title{
Sinomenine prevents the development of cardiomyopathy in diabetic rats by inhibiting inflammatory responses and blocking activation of NF-kB
}

\author{
Cheng Jiang, Yun-Long Tong, Dan Zhang, Li-Zhi Liu and Ju-Fei Wang \\ Department of Vasculocardiology, Fenghua people's hospital, 315500 Ningbo, China
}

\begin{abstract}
Diabetic cardiomyopathy is a severe complication of diabetes mellitus (DM). The goal of current work was to study the effects of sinomenine on streptozotocin-induced cardiomyopathy in rats. DM in rats was induced by intraperitoneal injection of streptozotocin. Cardiac function was evaluated by measuring left ventricle end-diastolic diameter, left ventricle end-systolic diameter and ejection fraction. Cardiac inflammation was evaluated by the degree of infiltration of Tlymphocytes and the levels of pro-inflammatory cytokines. Sinomenine attenuated diabetic symptoms without affecting plasma glucose. Cardiac dysfunction in the sinomenine-treated diabetic rats was significantly improved, as reflected by decreased levels of left ventricle end-diastolic diameter, left ventricle end systolic diameter and an increased level of ejection fraction. Sinomenine observably reduced cardiomyocyte hypertrophy in DM rats. Moreover, sinomenine reduced infiltration of $\mathrm{CD}^{+}$and $\mathrm{CD} 68^{+}$ positive cells and decreased the levels of tumor necrosis factor- $\alpha$, interlukin- 1 and interlukin- 6 . Finally, sinomenine-treated rats showed a reduced expression of NF- $\mathrm{KB}$ and an increased expression of IкB in the myocardium compared with the myocardium of untreated diabetic rats. Our results indicate sinomenine significantly improves cardiac function in diabetic rats, which may be attributed to the deactivation of NF- $\mathrm{kB}$ and the blockade of inflammatory cytokine-mediated immune reactions.
\end{abstract}

Key words: Sinomenine - Diabetic cardiomyopathy $-\mathrm{CD}^{+}-\mathrm{CD}^{+} 8^{+}-\mathrm{NF}-\mathrm{\kappa B}$

\section{Introduction}

Diabetic cardiomyopathy (DCM) is a severe complication of diabetes mellitus (DM) and is characterized by cardiac dysfunction and abnormal myocardial structure. DCM is independent of coronary artery disease and systemic hypertension (Sowers et al. 2001). As many lines of evidence (Zannad et al. 1999; From et al. 2006) have described previously, the prevalence of heart failure in DM patients varies from $12 \%$ to $48 \%$, and the incidence of DM patients with non-ischemic advanced heart failure is $19.7 \%$ (From et al. 2006). Notably, more than $70 \%$ of DM patients die from cardiovascular disease, and this ratio is 2-3-fold higher than the mortality by cardiovascular disease in non-diabetic patients (Boudina and Abel 2010; Zhang et al. 2012). A better understanding of the pathophysiology is a prerequisite for the development of new medicines. It is

Correspondence to: Dan Zhang, Park road no. 36 of Fenghua, 315500, Ningbo, China

E-mail: Zhangdanfh1985@163.com well-accepted that DCM is a multifactor illness, which involves the activation of the rennin-angiotensin system (RAS), oxidative stress, cardiac inflammation, cardiomyocyte apoptosis and interstitial fibrosis, all of which are activated by hyperglycemia (Boudina and Abel 2010; Zhang et al. 2012). In recent years, many studies have noted that cardiac inflammation may be core element of DCM. In DCM patients, hyperglycemia and dyslipidemia correlate with increased expression and secretion of cytokines. In turn, the levels of cytokines, such as tumor necrosis factor-a (TNF- $\alpha$ ) and interleukin-6 (IL-6), are closely associated with left ventricular diastolic dysfunction (Dint et al. 2009; Mahmoud and Al-Ozairi 2013). Furthermore, inflammation can signal to other pathways through cross talk that can also contribute to the formation of DCM via induction of oxidative stress and the activation of RAS. Etanercept (a well known TNF- $\alpha$ inhibitor) attenuates myocardial ischemia/ reperfusion injury by decreasing the inflammatory response and oxidative stress, while TNF- $\alpha$ itself induces apoptosis in rat cardiomyocytes and upregulates the angiotensin II type 1 receptor on cardiac fibroblasts (Gurantz et al. 2005; Yang et al. 
2014; Yu et al. 2014). In summary, these results suggest that active compounds with anti-inflammatory properties may be useful in preventing DCM.

Sinomenine (Sin, $\mathrm{C}_{19} \mathrm{H}_{23} \mathrm{NO}_{4}$, m.w. 329 D, Fig. 1) is an active alkaloid extracted from the root of the climbing plant Sinomenium acutum, known popularly as Chinese moonseed. It was firstly identified by Ishiwari in 1920 (Mayeda 1953). As reported previously, the compound possesses a diversified set of bioactivities, including anti-arrhythmic, antianginal, analgesic, anti-inflammatory and sedative activities (Zhao et al. 2012). In vivo studies, sinomenine effectively reduced the inflammatory response in animal models of arthritis and uveitis (Mu et al. 2013; Song et al. 2013). In an in vitro study, sinomenine blockaded activation of immune cells (T lymphocytes, dendritic cells, macrophages) and reduced production of pro-inflammatory cytokines (TNF- $\alpha$, IL-1) (Wang and Li 2011; Tong et al. 2015). Notably, a recent report demonstrated that sinomenine suppresses TNF- $\alpha$, IL- 1 and IL- 6 release in a dose-dependent fashion, and simultaneously reduces nuclear translocation of nuclear transcription factor-kappa B (NF- $\kappa \mathrm{B})$ in AGEsactivated retinal microglia in culture (Wang et al. 2007). Consequently, on the basis of these lines of evidence, we hypothesized that sinomenine may exert protective effects in DCM by suppressing myocardial inflammation and its downstream signaling targets.

\section{Materials and Methods}

\section{Animal experiments and treatment}

Ninety Wistar rats (180-220 g, male, six weeks old) were obtained from the animal department of Zhejiang University (Hangzhou, China). The animals were housed and allowed free access to a standard diet and tap water. The study was approved by the Animal Ethics Committee of Fenghua Peo-

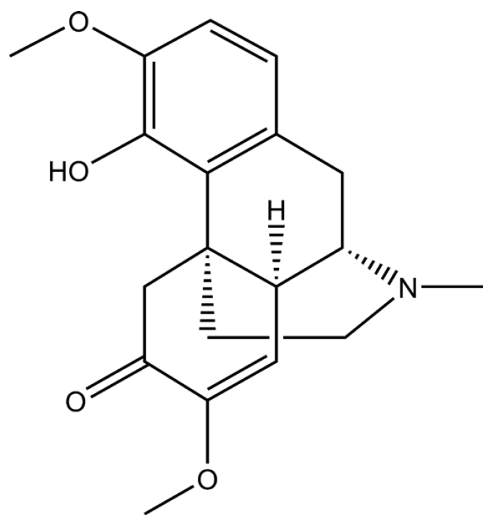

Figure 1. Chemical structure of sinomenine. ples Hospital (Fenghua, China). All the animal experiments were carried out according to the U.K. animals (Scientific Procedures) Act, 1986 and associated guidelines.

Diabetic rats were induced by a single intraperitoneal (i.p.) injection of streptozotocin (STZ, $60 \mathrm{mg} / \mathrm{kg}$, Sigma, St. Louis, MO, USA) in fresh $0.1 \mathrm{M}$ citrate buffer ( $\mathrm{pH}$ 4.5 , immediately used after preparation), while normal rats were injected with an equal volume of citrate buffer. The preparation of $0.1 \mathrm{M}$ citrate buffer was as following: Solution A was produced by dissolving $2.1 \mathrm{~g}$ citric acid into $100 \mathrm{ml}$ distilled water, and Solution B was formed by dissolving $2.94 \mathrm{~g}$ sodium citrate into $100 \mathrm{ml}$ distilled water; $0.1 \mathrm{M}$ citrate buffer was prepared by the mixture of solution $\mathrm{A}$ and solution $\mathrm{B}$ with a ratio of 1:1.32. Five days later, the STZ-treated rats $(n=80)$ with blood glucose levels $\geq 16.7 \mathrm{mM}$ were classified as being diabetic. Subsequently, these diabetic rats were randomly divided into five groups as follows: DM group, vehicle-treated rats; Sin30, Sin60, Sin 120 groups, diabetic rats treated with sinomenine at doses of 30, 60 and $120 \mathrm{mg} / \mathrm{kg}$; Los group, diabetic rats treated with losartan at dose of $30 \mathrm{mg} / \mathrm{kg}$ (sinomenine purity $>97 \%$, losartan purity $>99 \%$; Aladdin biological technology co., LTD, Shanghai, China). The appropriate vehicle or drug was administered orally to all the rats at the same time every day for ten weeks, following the induction of DM. The total incidence of induced DM in the rats was $81.25 \%(65 / 80)$, and during the experimental period, the mortality of the DM rats was $36.92 \%$ (41/65), which breaks down as follows: DM group (seven rats), Sin30 (seven rats), Sin60 (nine rats), Sin 120 (ten rats), Los group (eight rats). The normal control group contained ten rats.

\section{Measurement of cardiac function}

Ten weeks after drug administration, echocardiography was performed to evaluate the cardiac function of the diabetic rats. Briefly, rats were anesthetized with pentobarbital (i.p., $50 \mathrm{mg} / \mathrm{kg}$ ). Left ventricle end-diastolic diameter (LVEDD), left ventricle end systolic diameter (LVESD), diastolic interventricular septal wall thickness (IVSD), ejection fraction (EF) and heart rate (HR) were recorded using an echocardiography machine equipped with a $12-\mathrm{MHz}$ linear probe. The parameters were measured at least three times and then averaged.

The experimental animals were euthanized by $\mathrm{CO}_{2}$ inhalation, and then the hearts were removed and weighed. Heart weight (HW) and the ratio of HW to body weight (BW) were detected. Finally, the left ventricle was quickly cut into three parts. The first part was immediately stored at $-80^{\circ} \mathrm{C}$ for analysis of protein, the second part was used to measure production of pro-inflammatory cytokines, and the last part was fixed in $4 \%$ paraformaldehyde for histopathological and immunohistochemical study. 
Detection of plasma glucose

After evaluation of cardiac function, blood samples were drawn from the abdominal artery, and the concentrations of glucose were measured using a glucometer (Changsha Sinocare Inc. China).

\section{Histopathological and immunohistochemical studies}

The tissue was fixed in 4\% paraformaldehyde and embedded in paraffin, and $5-\mu \mathrm{m}$ sections were stained with hematoxylin-eosin. Pathological scores were recorded based on the cardiomyocyte hypertrophy in each slice (Yu et al. 2012). Cardiomyocyte hypertrophy was evaluated by the measurement of cells size, which is usually represented by the shortaxis diameters. The short-axis diameters were determined by the observation and measurement of the shortest diameters that passed through the cell nucleus. Short-axis diameters of cardiomyocytes were assayed for 10 myocytes per section at 400-fold magnification, using an inverted microscope (Olympus, Tokyo, Japan) equipped with an ocular micrometer, and then were averaged based on the data from 10 myocytes. For the study of immunohistochemistry (Wen et al. 2013), the sections were incubated with anti-CD68 ${ }^{+}$(Abcam, Cambridge, MA, USA) or anti-CD3 ${ }^{+}$(Abcam, Cambridge, MA, USA) antibodies overnight, and then treated with the corresponding secondary antibody for $30 \mathrm{~min}$. Ultimately, a DAB kit (Vectorlabs, USA) was used to color the sections, and the immunohistochemical staining was quantified by Image Pro Plus on 10 fields for the left ventricle.

\section{Measurement of pro-inflammatory cytokines in cardiac tissue}

For detection of inflammatory cytokines, the cardiac tissue was cut into pieces and then homogenized and lysed. After centrifugation at $16000 \times g$ for $10 \mathrm{~min}\left(4^{\circ} \mathrm{C}\right)$, the supernatants was collected and stored at $-80^{\circ} \mathrm{C}$ for further analysis. The levels of TNF- $\alpha$, IL- 1 and IL- 6 in supernatants were determined by commercial ELISA kits (Invitrogen, Camarillo, CA).

\section{Western blot analysis}

After rinsing with PBS, heart tissue was treated with lysis buffer. Following a centrifugation step $(12000 \times g, 15 \mathrm{~min})$, the supernatant was removed and stored at $-80^{\circ} \mathrm{C}$ for protein analysis. Subsequently, $50 \mu \mathrm{g}$ of extracted protein was loaded and separated by SDS-PAGE followed by electrophoretic transfer to nitrocellulose filters. The membranes were blocked with 5\% non-fat dry milk and incubated with different primary antibodies at $4^{\circ} \mathrm{C}$ overnight. The following antibodies were used in this study: I $\kappa B$ and NF- $\kappa B$ p 65 (Abcam, Cambridge, MA, USA). After incubation with the secondary antibody, the blots were visualized using an ECL kit (Pierce Biosciences, Billerica, MA, USA).

\section{Statistical analysis}

The results are expressed as the means \pm SD. SPSS (version 14.0) software was used to perform the statistical analysis. Oneway analysis of variance (ANOVA) and Dunnett's t-test were used to assess the significance of any change between groups. A value of $p<0.05$ was considered to be statistically significant.

\section{Results}

Sinomenine reduces STZ-induced diabetic pathology without affecting plasma glucose

In the DM group, STZ-induced diabetic rats exhibited markedly lower body weight and higher blood glucose level compared with the negative control (NC) group $(p<0.01$; Fig. 2). However, administration of sinomenine to diabetic
A

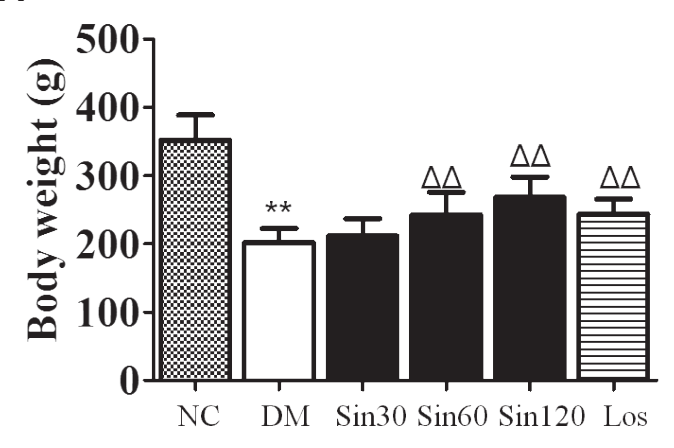

B

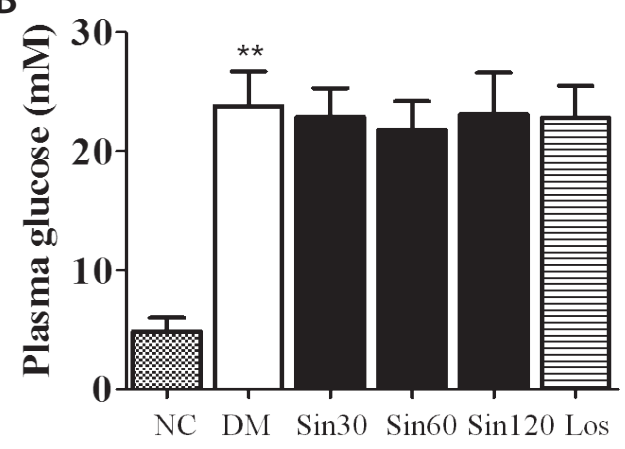

Figure 2. Effect of sinomenine on diabetic performance in experimental diabetic rats. At the end of study, the body weights of the mice were measured (A) and the blood samples were collected from abdominal artery for the plasma glucose detection (B). The data are presented as the mean $\pm \mathrm{SD}, n=$ $7-10 ;{ }^{* *} p<0.01$ vs. NC

group; ${ }^{\Delta \Delta} p<0.01$ vs. DM group. NC, normal control group; DM, group of rats with induced diabetes mellitus; $\operatorname{Sin} 30$, Sin60, Sin120, diabetic rats treated with sinomenine at doses of 30,60 and $120 \mathrm{mg} / \mathrm{kg}$; Los, diabetic rats treated with losartan at dose of $30 \mathrm{mg} / \mathrm{kg}$. 
rats strikingly increased their body weights compared with the DM group $(p<0.01)$. In addition, sinomenine had no effect on the level of blood glucose. We also found that Lostreated diabetic rats showed similar results to those observed with sinomenine.

\section{Sinomenine prevents diabetic-induced cardiac dysfunction}

As shown in Table 1 and Figure 3H, in the DM group, STZinduced diabetic rats exhibited significant cardiac dysfunction, as demonstrated by increased values of IVSD, LVEDD, LVESD, cardiac index, and decreased values of heart rate and $\mathrm{EF}(p<0.01)$. However, oral administration of sinomenine to the diabetic rats caused decreases in IVSD, LVEDD, LVESD and cardiac index and increases in the heart rate and EF in comparison to the DM group $(p<0.05)$. These findings suggest sinomenine could improve diabetic induced-cardiac dysfunction.

\section{Sinomenine improves cardiac histopathology in diabetic rats}

To evaluate the cardioprotective effects of sinomenine further, myocardial structural was analyzed by H\&E staining. Perinuclear vacuolization occurred in the DM rats (Fig. 3B) but this property was normalized in sinomenine-treated rats (Fig. 3D,E). Additionally, cell size was measured to evaluate the cardiomyocyte hypertrophy of the left ventricle. As can be seen in Fig. 3G, cardiomyocyte diameters in the DM group were obviously greater than those in the normal group $(p<0.01)$. Notably, in sinomenine-treated diabetic rats, cardiomyocyte hypertrophy was clearly reduced compared with those in the DM group $(p<0.05)$. These results indicate definitively that sinomenine can ameliorate cardiac histopathology in diabetic rats.
Sinomenine inhibits cardiac inflammation in STZ-induced diabetic rats

Previous studies (Yu et al. 2012; Wen et al. 2013) demonstrated that impaired cardiac function is closely associated with cardiac inflammatory responses. To further disclose the potential mechanism of sinomenine action, we focused on the effects of sinomenine on cardiac infiltration of $\mathrm{T}$ lymphocytes and macrophages and on the production of pro-inflammatory cytokines in diabetic rats. As seen in Figure 4 , in the DM group, the numbers of $\mathrm{CD}^{+}$( $\mathrm{T}$ lymphocyte, Fig. 4B) and CD $68^{+}$positive cells (macrophage, Fig. 4E) were significantly higher than those in the normal group $(p<0.01)$. However, sinomenine markedly reduced infiltration of these cells in diabetic rats $(p<0.01)$. Moreover, the levels of TNF-a, IL-1 and IL-6 in diabetic myocardium were reduced significantly after oral administration of sinomenine (Fig. 5; $p<0.01$ ). These results revealed that sinomenine could reduce cardiac inflammation in STZinduced diabetic rats.

\section{Sinomenine prevents the activation of $N F-\kappa B$ in diabetic rats}

It is well-known that cross-talk between pathways can occur through NF- $\kappa \mathrm{B}$, which is a key point of intersection of several cytokine-mediated pathways that are involved in amplification of the inflammatory response (Lorenzo et al. 2011). To uncover possible mechanisms by which sinomenine inhibits cardiac inflammation, we measured cardiac expression of NF- $\kappa \mathrm{B}$ and $\mathrm{I} \kappa \mathrm{B}$ in the various groups. Figure 6 presents the decreased expression of I $\kappa \mathrm{B}$ and increased expression of NF- $\kappa \mathrm{B}$ in the diabetic rats, indicative of activated NF- $\kappa \mathrm{B}$ signaling; this is consistent with the result reported previously (Wang et al. 2005). Strikingly, sinomenine abolished cardiac activation of NF- $\mathrm{BB}$ in the diabetic rats $(p<0.01)$.

Table 1. Sin alleviated STZ induced left ventricular dysfunction in diabetic rats

\begin{tabular}{llllll}
\hline Group & $\begin{array}{c}\text { Heart rate } \\
(\mathrm{bpm})\end{array}$ & $\begin{array}{c}\text { IVSD } \\
(\mathrm{mm})\end{array}$ & $\begin{array}{c}\text { LVEDD } \\
(\mathrm{mm})\end{array}$ & $\begin{array}{c}\text { LVESD } \\
(\mathrm{mm})\end{array}$ & $\begin{array}{c}\text { EF } \\
(\%)\end{array}$ \\
\hline NC & $376 \pm 14$ & $1.37 \pm 0.09$ & $2.82 \pm 0.17$ & $1.50 \pm 0.08$ & $84.6 \pm 2.2$ \\
DM & $264 \pm 9^{* *}$ & $1.97 \pm 0.14^{* *}$ & $3.40 \pm 0.28^{* *}$ & $2.27 \pm 0.16^{* *}$ & $71.4 \pm 3.9^{* *}$ \\
Sin30 & $288 \pm 13^{\Delta}$ & $1.84 \pm 0.13$ & $3.31 \pm 0.22$ & $2.12 \pm 0.13$ & $74.8 \pm 3.1$ \\
Sin60 & $306 \pm 17^{\Delta \Delta}$ & $1.72 \pm 0.12^{\Delta \Delta}$ & $3.17 \pm 0.19^{\Delta}$ & $1.98 \pm 0.19^{\Delta}$ & $76.2 \pm 3.3^{\Delta \Delta}$ \\
Sin120 & $325 \pm 15^{\Delta \Delta}$ & $1.55 \pm 0.16^{\Delta \Delta}$ & $3.01 \pm 0.20^{\Delta \Delta}$ & $1.77 \pm 0.22^{\Delta \Delta}$ & $82.3 \pm 3.5^{\Delta \Delta}$ \\
Los & $338 \pm 19^{\Delta \Delta}$ & $1.40 \pm 0.11^{\Delta \Delta}$ & $3.02 \pm 0.13^{\Delta \Delta}$ & $1.80 \pm 0.12^{\Delta \Delta}$ & $80.4 \pm 2.4^{\Delta \Delta}$ \\
\hline
\end{tabular}

Data are presented as means \pm SD, $n=7-10 .^{* *} p<0.01 v s$. NC group; ${ }^{\Delta} p<0.05,{ }^{\Delta \Delta} p<0.01 v s$. DM group. IVSD, diastolic interventricular septal wall thickness; LVEDD, left ventricle end-diastolic diameter; LVESD, left ventricle end systolic diameter; EF, ejection fraction = [(LVEDD3- LVESD3)/LVEDD3] × 100\%; NC, normal control group; DM, group of rats with induced diabetes mellitus; Sin30, Sin60, Sin120, diabetic rats treated with sinomenine at doses of 30, 60 and $120 \mathrm{mg} / \mathrm{kg}$; Los, diabetic rats treated with losartan at dose of $30 \mathrm{mg} / \mathrm{kg}$. 

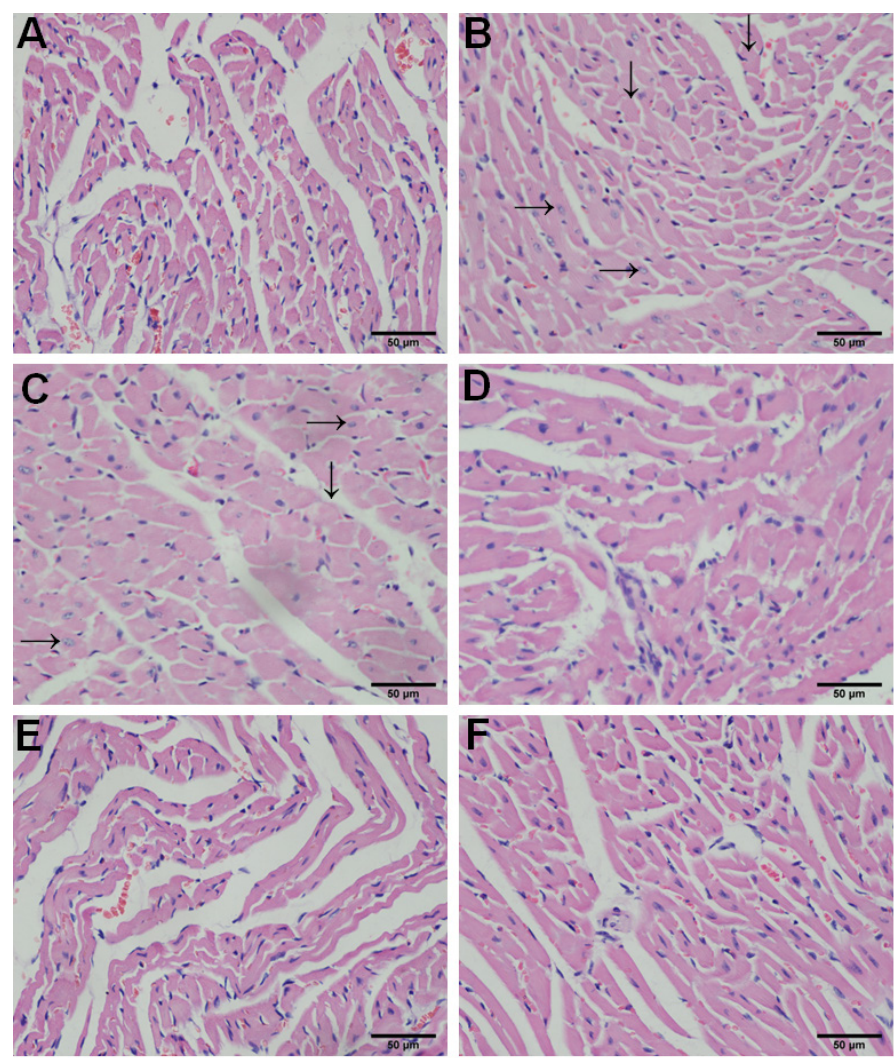

G

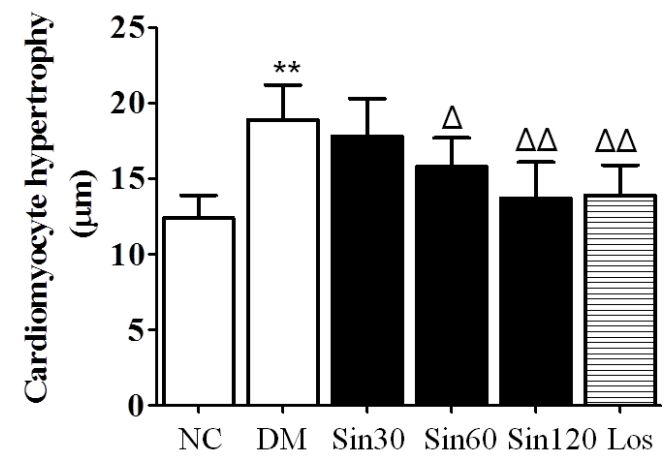

H

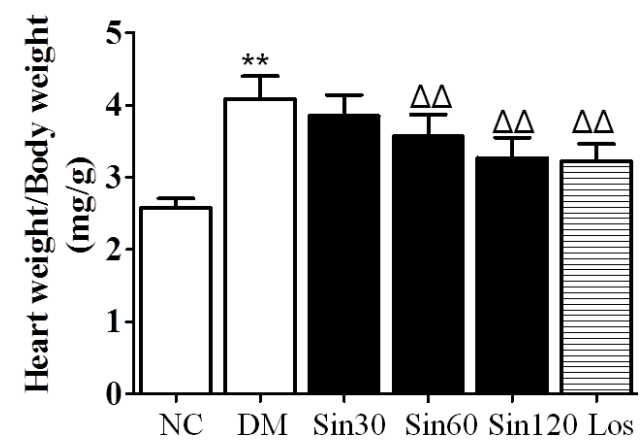

Figure 3. Effect of sinomenine on cardiac histopathology in experimental diabetic rats. A. Cardiac tissue of normal rats. B. Cardiac tissue in DM rats exhibits significant perinuclear vacuolization (horizontal arrow) and cardiomyocyte hypertrophy (vertical arrow). Cardiac tissue in $\operatorname{Sin} 30$ (C), Sin60 (D), Sin120 (E), and Los (F) groups. The indicated scales in the pictures are $50 \mu \mathrm{m}$. G. Quantitative analysis of cardiomyocyte diameter. H. Cardiac index as determined by the value of heart weight/body weight. Data are presented as the mean $\pm \mathrm{SD}, n=7-10 ;{ }^{* *} p<0.01$ vs. NC group; ${ }^{\Delta} p<0.05,{ }^{\Delta \Delta} p<0.01$ vs. DM group. (For abbreviations, see Fig. 1).

\section{Discussion}

Many reports have confirmed that myocardial inflammation is the main contributor to DCM. In diabetic patients, increased production of inflammatory cytokines is associated with diastolic dysfunction (Hoffman et al. 2013), and severe, reversible cardiomyopathy is associated with systemic inflammatory response syndrome in the setting of diabetic, hyperglycemic, non-ketotic syndrome (Berk et al. 2015). Moreover, hyperglycemia-induced myocardial damage is mediated both by pro-inflammatory cytokines and by macrophage migration inhibitory factor (Yu et al. 2011). In diabetic animal models, many reports have confirmed the enhanced infiltrations of T lymphocytes and macrophages, and the increased production of pro-inflammatory cytokines (TNF- $\alpha$, IL-1 and IL-6) in the myocardium (Yu et al. 2012; Wen et al. 2013). It is widely believed that myocardial inflammation in DCM is originally induced by hyperglycemia (Pan et al. 2014). Subsequently, inflammatory factors maybe disrupt cardiac function directly as demonstrated by the following observations. High levels of IL-1, IL-6 and TNF- $\alpha$ directly inhibit rat cardiomyocyte contractility in vitro (Hobai et al. 2015). TNF-a provokes a concentric cardiac hypertrophic phenotype, induces apoptosis of cardiac myocytes, and contributes to progressive left ventricular wall thinning and adverse cardiac remodeling (Bozkurt et al. 1998; Dibbs et al. 2003; Haudek et al. 2007). Mice with cardiac-specific over-expression of IL-1 show concentric left ventricular hypertrophy with preserved left ventricular systolic function. IL-1 also induces programmed cell death in cultured cardiac myocytes through NOS induction (Ing et al. 1999; Nishikawa et al. 2006). Regarding IL-6, continuous over-expression of both IL-6 and the IL-6 receptor (IL-6R) in double transgenic mice is associated with cardiac myocyte hypertrophy. However, neither IL-6 nor IL-6R over-expression alone is sufficient to induce detectable myocardial abnormalities due to the low expression level of IL-6R in cardiac myocytes (Saito et al. 1992; Hirota et al. 1995).

It is well-known that cytokines mediate cardiac inflammation via downstream activation of NF- $\kappa \mathrm{B}$. Under physi- 

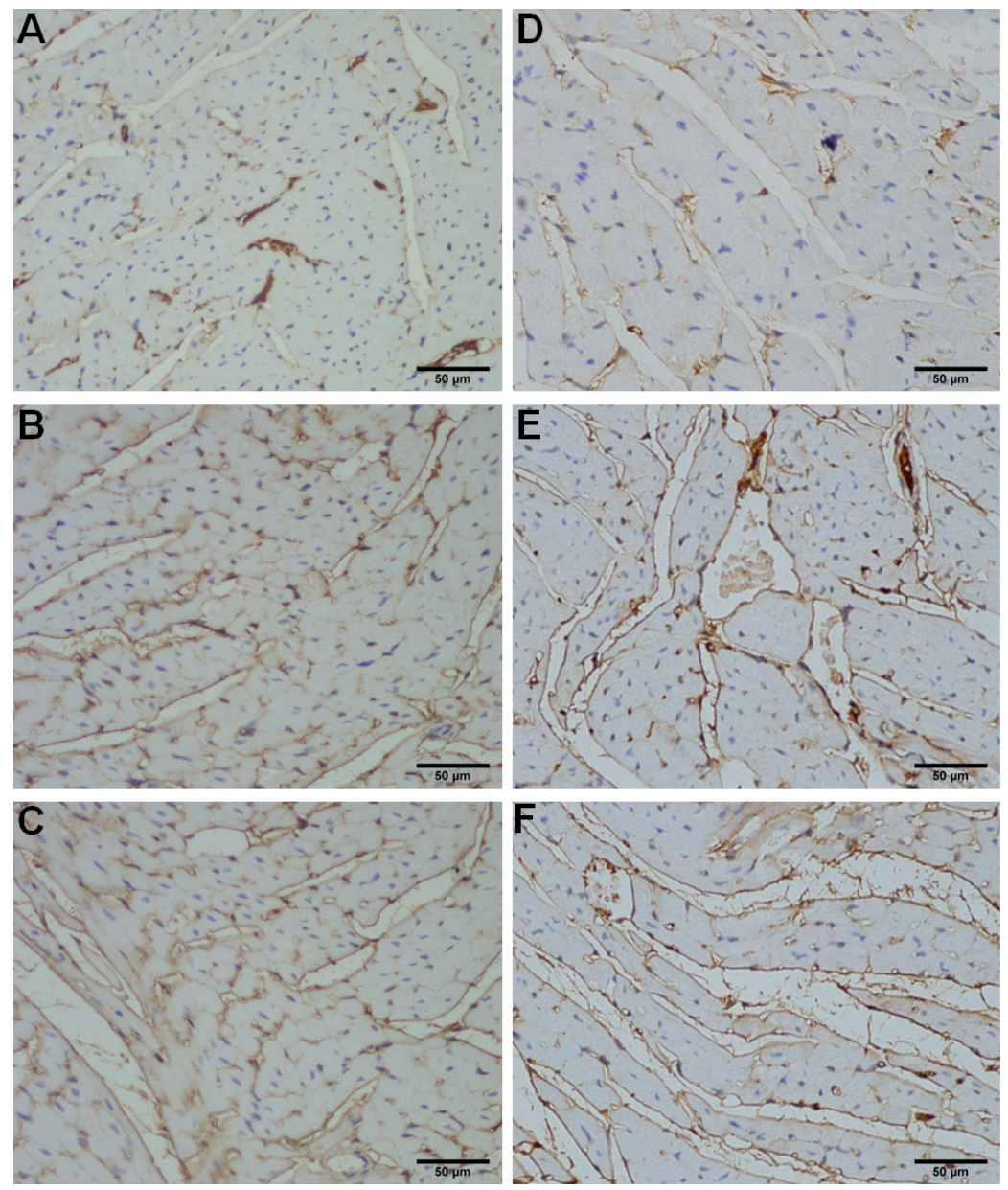

\section{G}
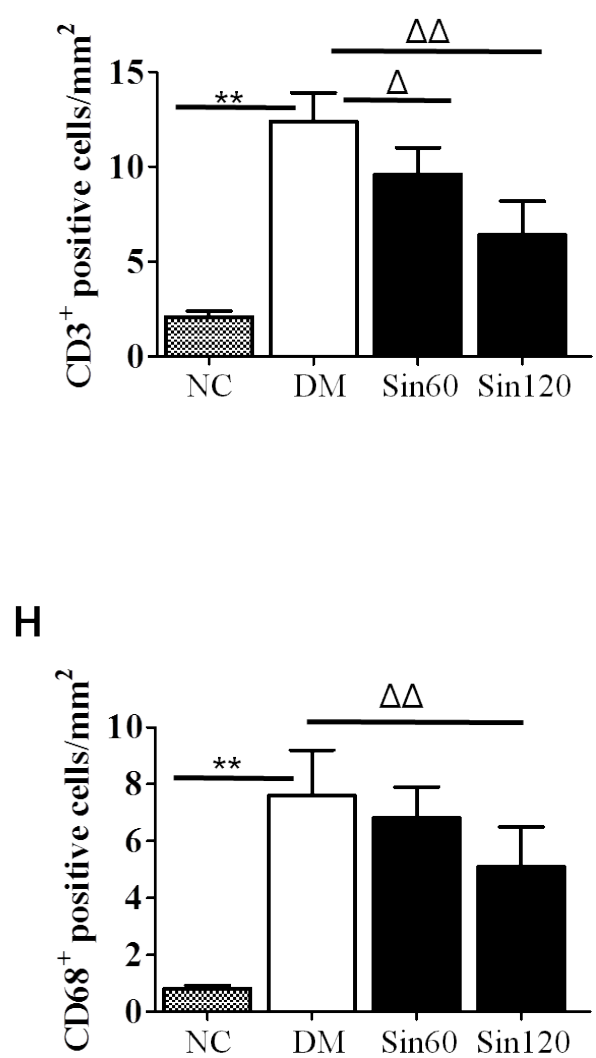

Figure 4. Effect of sinomenine on inflammatory cells infiltration in cardiac tissue of diabetic rats. A. CD $3^{+}$positive cells in normal rats. B. $\mathrm{CD}^{+}$positive cells in DM rats. C. $\mathrm{CD}^{+}$positive cells in Sin 120 group. D. $\mathrm{CD} 68^{+}$positive cells in normal rats. E. $\mathrm{CD} 68^{+}$positive cells in DM rats. F. D68 ${ }^{+}$positive cells in Sin 120 group. The indicated scales in the pictures are $50 \mu \mathrm{m}$. G. Quantitative analysis of CD $3^{+}$ positive cells numbers. H. Quantitative analysis of $\mathrm{CD} 68^{+}$positive cells numbers. Data are presented as the mean $\pm \mathrm{SD}, n=7-10$; ${ }^{* *} p<$ $0.01 v s$. NC group; ${ }^{\Delta} p<0.05,{ }^{\Delta \Delta} p<0.01 v$ s. DM group. (For abbreviations, see Fig. 1).

ological conditions, NF- $\mathrm{KB}$ is bound to its inhibitory proteins, known as IkBs (Lorenzo et al. 2011). When the cell receives a pathological stimulus, such as high glucose, oxidative stress or production of pro-inflammatory cytokines, $\mathrm{NF}-\mathrm{\kappa B}$ will be activated by dissociation from I $\kappa$ B. Subsequently, the activated form of NF- $\kappa \mathrm{B}$ will translocate into the nucleus, bind to NF- $\kappa \mathrm{B}$-consensus sequences in genomic DNA (named $\kappa \mathrm{B}$ sites) and thereby regulate gene transcription (Lorenzo et al. 2011). Of note, the expression levels of almost 200 target genes have been reported to be modulated by NF- $\kappa B$, and most of them are involved in the modulation of inflammatory responses (Hall et al. 2006; Lorenzo et al. 2011). Interestingly, activation of NF- $\kappa \mathrm{B}$ induces transcription of inflammatory cytokines, and the released cytokines can bind with their receptors, which results in further activation of NF- $\kappa \mathrm{B}$ signaling. As reported previously, when cardiomyocytes are treated with a TNF- $\alpha$ antagonist (etanercept), they shows decreased levels of NF- $\kappa B$ signaling ( $\mathrm{Xu}$ et al. 2008), and in TNF- $\alpha$ receptor deficient mice, NF- $\kappa \mathrm{B}$ activation is greatly suppressed when compared to wild-type mice (Aoki et al. 2014). Therefore, overactivation of inflammatory cytokines and of NF- $\kappa B$ results in a vicious spiral instead of a classical negative feedback.

The role of NF- $\kappa \mathrm{B}$ in DCM is well-established. As discussed previously, in patients with myocarditis and dilated cardiomyopathy, activation of NF- $\mathrm{BB}$ closely correlates with left ventricular function (Alter et al. 2006). In animal models, cardiomyocyte over-expression of $\mathrm{I} \kappa \mathrm{B}$ (inhibitor of NF- $\kappa \mathrm{B}$ ) attenuated TNF- $\alpha$-induced fractional shortening and ventricular hypertrophy (Higuchi et al. 2006), and blockade of NF- $\kappa \mathrm{B}$ activation improved cardiac function and survival in male TNF-transgenic mice (Kawamura et al. 2005). 
A

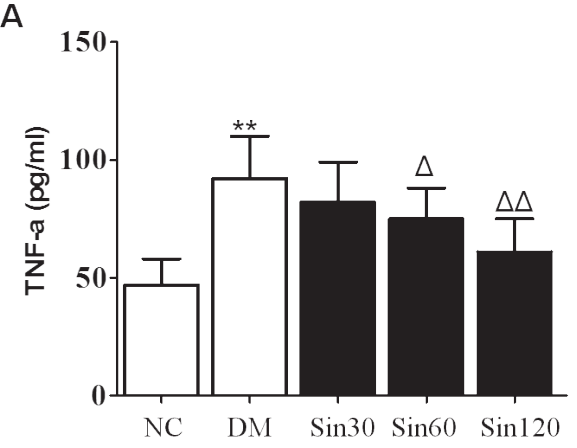

B

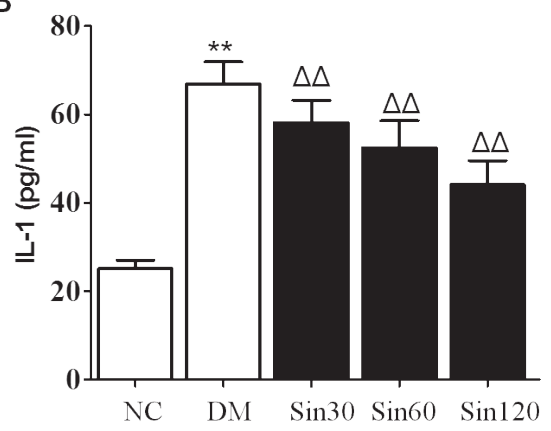

C

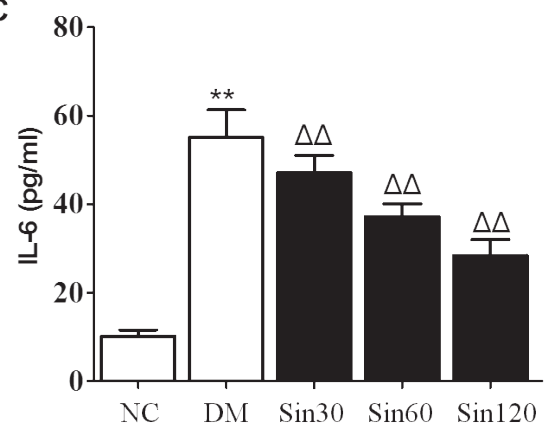

Figure 5. Effect of sinomenine on pro-inflammatory cytokines levels in cardiac tissue of diabetic rats. A. TNF- $\alpha$. B. IL-1. C. IL-6. Data are presented as the mean $\pm \mathrm{SD}, n=5 ;{ }^{* *} p<0.01 v s$. NC group; ${ }^{\Delta} p<0.05,{ }^{\Delta \Delta} p<0.01$ vs. DM group. IL-1, interlukin-1; TNF- $\alpha$, tumor necrosis factor- $\alpha$. (Other abbreviations, see Fig. 1).

Therefore, it is proposed that inhibition of NF- $\mathrm{B}$ may be a useful strategy for preventing DCM, especially when proinflammatory cytokines are overactivated.

Although the role of pro-inflammatory cytokines is clear, the results of large multicenter trials of etanercept in moderate to severe heart failure did not demonstrate any clinical benefits and may adversely affect the course of the disease (Anker and Coats 2002; Coletta et al. 2002). It is widely believed that the disordered cytokines networks in DCM are complex, and strict control of one or several cytokines is insufficient to inhibit the myocardial lesions. Sinomenine,
A

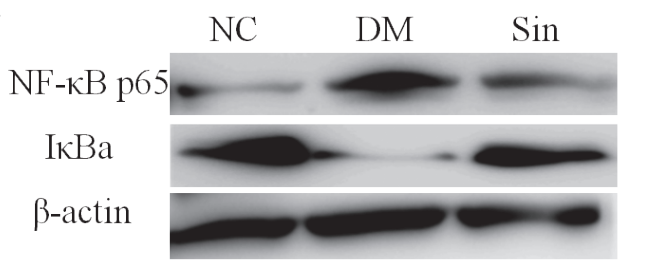

B

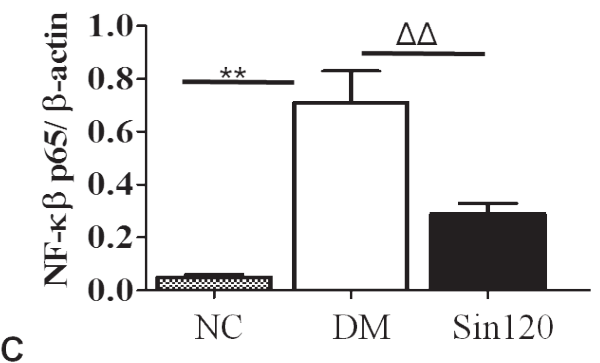

C

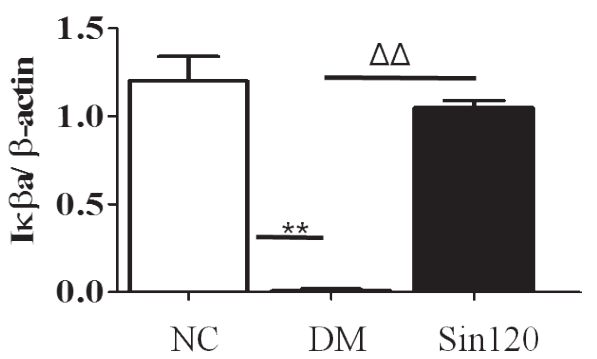

Figure 6. Effect of sinomenine on NF- $\mathrm{BB}$ signaling in diabetic rats. A. Representative lanes of targeted proteins. B. Western blot analysis of NF- $\kappa \mathrm{B}$ p65. C. Western blot analysis of IкB. Data are presented as the mean $\pm \mathrm{SD}, n=5 ;{ }^{* *} p<0.01 v$ s. NC group; ${ }^{\Delta \Delta} p<$ 0.01 vs. DM group. (For abbreviations, see Fig. 1).

first discovered in 1920, has been confirmed to possess antiinflammatory activity. In the past 30 years, the therapeutic efficacy and lower side effects of purified sinomenine in patients with rheumatoid arthritis and nephritis have been confirmed in open clinical trials (Cheng et al. 2009; Wang and $\mathrm{Li}$ 2011). In recent years, the anti-inflammatory mechanism of sinomenine has been uncovered. At the cellular level, sinomenine reduces proliferation of lymphocytes, keeps $\mathrm{T}$ lymphocyte subtypes balanced, reduces the invasion and migration ability of monocytes, prevents DC maturity and inhibits DC antigen presentation (Zhao et al. 2007; Cheng et al. 2009; Tong 2015). At the molecular level, sinomenine attenuates nuclear translocation of the NF- $\kappa \mathrm{B}$ p 65 subunit and the DNA-binding activity of NF- $\kappa \mathrm{B}$ (Wang et al. 2007; Cheng et al. 2009; Zhao et al. 2013). In addition, sinomenine also reduces the production of IL- $1 \beta$ and TNF- $\alpha$ in synovial cells of rats with adjuvant-induced arthritis (Anker and Coats 2002; Zhao et al. 2012; Mu et al. 2013; Zhao et al. 2013).

In this work, it is firstly discovered that treatment of sinomenine significantly improves cardiac function in diabetic rats, as evidenced by the increases in the heart rate and 
EF, and by decreased values of IVSD, LVEDD and LVESD. Of note, when comparing the Los group and the Sin 120 group, it seems that losartan is more effective in improving heart rate and the IVSD, whereas sinomenine is more effective in improving LVEDD, LVESD and EF. However, after careful analysis, we confirmed that there were no significant differences in the cardioprotective effects between the groups treated with losartan or sinemonine. Therefore, it can be concluded that Sin 120 and Los groups possess the same pharmacologic effects in the prevention of ventricular dysfunction. Interestingly, sinomenine did not alter plasma glucose, which suggests the cardiovascular protective effects are independent of anti-diabetic activity. Moreover, it is worth mentioning that a portion of DCM patients have symptomatic features, such as hypotension, and losartan may not be a suitable drug for these patients (Bellmann and Tschöpe 2014; Dimitropoulos et al. 2014). Furthermore, angiotensin receptor blockers easily damage renal function in some special classes of patients, including the elderly, children, and heart failure and renal artery stenosis patients (Maillard et al. 2001; Kiykim et al. 2004). Therefore, for these patients, sinomenine may be provided as an alternative medication, based on its safety and efficacy. Finally, further research showed that sinomenine observably inhibited infiltration of $\mathrm{T}$ lymphocytes and macrophages, reduced the levels of pro-inflammatory cytokines (TNF- $\alpha$, IL-1 and IL-6) and blocked cardiac activation of NF- $\kappa$ B. Our results showed that sinomenine therapy can undermine cardiac inflammatory responses and deactivate the NF- $\kappa B$ signaling pathway.

\section{Conclusions}

Taken together, the findings of the present study demonstrate that sinomenine improves cardiac function in DM rats. Sinomenine preserves the myocardium by reducing NF- $\kappa \mathrm{B}$ activation and inhibiting the production of proinflammatory cytokines that mediate myocardial inflammation.

Acknowledgements. This study was financially supported by the technology projects of Fenghua city (Grant No. 201508809).

Conflict of interest. All authors have approved the final article, and declare that they have no conflict of interest.

\section{References}

Alter P., Rupp H., Maisch B. (2006): Activated nuclear transcription factor kappaB in patients with myocarditis and dilated cardiomyopathy-relation to inflammation and cardiac function. Biochem. Biophys. Res. Commun. 339, 180-187 https:/doi.org/10.1016/j.bbrc.2005.10.195
Anker S. D., Coats A. J. (2002): How to recover from renaissance? The significance of the results of recover, renaissance, renewal and attach. Int. J. Cardiol. 86, 123-130 https:/doi.org/10.1016/S0167-5273(02)00470-9

Aoki T., Fukuda M., Nishimura M., Nozaki K., Narumiya S. (2014): Critical role of TNF-alpha-TNFR1 signaling in intracranial aneurysm formation. Acta Neuropathol. Commun. 2, 34 https:/doi.org/10.1186/2051-5960-2-34

Bellmann B., Tschöpe C. (2014): Heart failure. Cardiovascular autonomic neuropathy in patients with diabetes mellitus. Herz 39, 306-311 (in German) https:/doi.org/10.1007/s00059-014-4093-2

Berk J., Wade R., Baser H. D., Lado J. (2015): Case report: severe reversible cardiomyopathy associated with systemic inflammatory response syndrome in the setting of diabetic hyperosmolar hyperglycemic non-ketotic syndrome. BMC Cardiovasc. Disord. 15, 123 https:/doi.org/10.1186/s12872-015-0112-3

Boudina S., Abel E. D. (2010): Diabetic cardiomyopathy, causes and effects. Rev. Endocr. Metab. Disord. 11, 31-39 https:/doi.org/10.1007/s11154-010-9131-7

Bozkurt B., Kribbs S. B., Clubb F. J. Jr., Michael L. H., Didenko V. V., Hornsby P. J., Seta Y., Oral H., Spinale F. G., Mann D. L. (1998): Pathophysiologically relevant concentrations of tumor necrosis factor-alpha promote progressive left ventricular dysfunction and remodeling in rats. Circulation 97, 1382-1391 https:/doi.org/10.1161/01.CIR.97.14.1382

Cheng Y., Zhang J., Hou W., Wang D., Li F., Zhang Y., Yuan F. (2009): Immunoregulatory effects of sinomenine on the T-bet/ GATA-3 ratio and Th1/Th2 cytokine balance in the treatment of mesangial proliferative nephritis. Int. Immunopharmacol. 9, 894-899 https:/doi.org/10.1016/j.intimp.2009.03.014

Coletta A. P., Clark A. L., Banarjee P., Cleland J. G. (2002): Clinical trials update: RENEWAL (RENAISSANCE and RECOVER) and ATTACH. Eur. J. Heart Fail. 4, 559-561 https:/doi.org/10.1016/S1388-9842(02)00121-6

Dibbs Z. I., Diwan A., Nemoto S., DeFreitas G., Abdellatif M., Carabello B. A., Spinale F. G., Feuerstein G., Sivasubramanian N., Mann D. L. (2003): Targeted overexpression of transmembrane tumor necrosis factor provokes a concentric cardiac hypertrophic phenotype. Circulation 108, 1002-1008 https:/doi.org/10.1161/01.CIR.0000085203.46621.F4

Dimitropoulos G., Tahrani A. A., Stevens M. J. (2014): Cardiac autonomic neuropathy in patients with diabetes mellitus. World J. Diabetes 5, 17-39 https:/doi.org/10.4239/wjd.v5.i1.17

Dint W., Füth R., Nickl W., Krahn T., Ellinghaus P., Scheffold T., Bansemir L., Bufe A., Barroso M. C., Lankisch M. (2009): Elevated plasma levels of TNF-alpha and interleukin-6 in patients with diastolic dysfunction and glucose metabolism disorders. Cardiovasc. Diabetol. 8, 58 https:/doi.org/10.1186/1475-2840-8-58

From A. M., Leibson C. L., Bursi F., Redfield M. M., Weston S. A., Jacobsen S. J., Rodeheffer R. J., Roger V. L. (2006): Diabetes in heart failure: prevalence and impact on outcome in the population. Am. J. Med. 119, 591-599 https:/doi.org/10.1016/j.amjmed.2006.05.024 
Gurantz D., Cowling R. T., Varki N., Frikovsky E., Moore C. D., Greenberg B. H. (2005): IL-1beta and TNF-alpha upregulate angiotensin II type 1 (AT1) receptors on cardiac fibroblasts and are associated with increased AT1 density in the post-MI heart. J. Mol. Cell. Cardiol. 38, 505-515 https:/doi.org/10.1016/j.yjmcc.2004.12.015

Hall G., Hasday J. D., Rogers T. B. (2006): Regulating the regulator: NF-kappaB signaling in heart. J. Mol. Cell. Cardiol. 41, 580-591 https:/doi.org/10.1016/j.yjmcc.2006.07.006

Haudek S. B., Taffet G. E., Schneider M. D., Mann D. L. (2007): TNF provokes cardiomyocyte apoptosis and cardiac remodeling through activation of multiple cell death pathways. J. Clin. Invest. 117, 2692-2701 https:/doi.org/10.1172/JCI29134

Higuchi Y., Chan T. O., Brown M. A., Zhang J., DeGeorge B. R. Jr., Funakoshi H., Gibson G., McTiernan C. F., Kubota T., Jones W. K., Feldman A. M. (2006): Cardioprotection afforded by NF-kappaB ablation is associated with activation of Akt in mice overexpressing TNF-alpha. Am. J. Physiol. Heart Circ. Physiol. 290, H590-598 https:/doi.org/10.1152/ajpheart.00379.2005

Hirota H., Yoshida K., Kishimoto T., Taga T. (1995): Continuous activation of gp130, a signal-transducing receptor component for interleukin 6-related cytokines, causes myocardial hypertrophy in mice. Proc. Natl. Acad. Sci. USA 92, 4862-4866 https:/doi.org/10.1073/pnas.92.11.4862

Hobai I. A., Morse J. C., Siwik D. A., Colucci W. S. (2015): Lipopolysaccharide and cytokines inhibit rat cardiomyocyte contractility in vitro. J. Surg. Res. 193, 888-901 https:/doi.org/10.1016/j.jss.2014.09.015

Hoffman W. H., Passmore G. G., Hannon D. W., Talor M. V., Fox P., Brailer C., Haislip D., Keel C., Harris G., Rose N. R., Fiordalisi I., Čiháková D. (2013): Increased systemic Th17 cytokines are associated with diastolic dysfunction in children and adolescents with diabetic ketoacidosis. PLoS One 8, e71905 https:/doi.org/10.1371/journal.pone.0071905

Ing D. J., Zang J., Dzau V. J., Webster K. A., Bishopric N. H. (1999):Modulation of cytokine-induced cardiac myocyte apoptosis by nitric oxide, Bak, and Bcl-x. Circ. Res. 84, 21-33 https:/doi.org/10.1161/01.RES.84.1.21

Kawamura N., Kubota T., Kawano S., Monden Y., Feldman A. M., Tsutsui H., Takeshita A., Sunagawa K. (2005): Blockade of NF-kappaB improves cardiac function and survival without affecting inflammation in TNF-alpha-induced cardiomyopathy. Cardiovasc. Res. 66, 520-529 https:/doi.org/10.1016/j.cardiores.2005.02.007

Kiykim A. A., Boz M., Ozer C., Camsari A., Yildiz A. (2004): Two episodes of anuria and acute pulmonary edema in a losartantreated patient with solitary kidney. Heart Vessels 19, 52-54 https:/doi.org/10.1007/s00380-003-0723-y

Lorenzo O., Picatoste B., Ares-Carrasco S., Ramírez E., Egido J., Tu-ón J. (2011): Potential role of nuclear factor $\kappa \mathrm{B}$ in diabetic cardiomyopathy. Mediators Inflamm. 2011, 652097 https:/doi.org/10.1155/2011/652097

Mahmoud F., Al-Ozairi E. (2013): Inflammatory cytokines and the risk of cardiovascular complications in type 2 diabetes. Dis. Markers 35, 235-241

https:/doi.org/10.1155/2013/931915
Maillard J. O., Descombes E., Fellay G., Regamey C. (2001): Repeated transient anuria following losartan administration in a patient with a solitary kidney. Ren. Fail. 23, 143-147 https:/doi.org/10.1081/JDI-100001295

Mayeda H. (1953): The release of histamine by sinomenine. Jpn. J. Pharmacol. 3, 62-72

https:/doi.org/10.1254/jjp.3.62

Mu H., Yao R. B., Zhao L. J., Shen S. Y., Zhao Z. M., Cai H. (2013): Sinomenine decreases MyD88 expression and improves inflammation-induced joint damage progression and symptoms in rat adjuvant-induced arthritis. Inflammation 36, 1136-1144 https:/doi.org/10.1007/s10753-013-9648-5

Nishikawa K., Yoshida M., Kusuhara M., Ishigami N., Isoda K., Miyazaki K., Ohsuzu F. (2006): Left ventricular hypertrophy in mice with a cardiac-specific overexpression of interleukin-1. Am. J. Physiol. Heart Circ. Physiol. 291, H176-183 https:/doi.org/10.1152/ajpheart.00269.2005

Pan Y., Wang Y., Zhao Y., Peng K., Li W., Wang Y., Zhang J., Zhou S., Liu Q., Li X., Cai L., Liang G. (2014): Inhibition of JNK phosphorylation by a novel curcumin analog prevents high glucose-induced inflammation and apoptosis in cardiomyocytes and the development of diabetic cardiomyopathy. Diabetes 63, 3497-3511 https:/doi.org/10.2337/db13-1577

Saito M., Yoshida K., Hibi M., Taga T., Kishimoto T. (1992): Molecular cloning of a murine IL-6 receptor-associated signal transducer, gp130, and its regulated expression in vivo. J. Immunol. 148, 4066-4071

Song J., Bi H., Xie X., Guo J., Wang X., Liu D. (2013): Preparation and evaluation of sinomenine hydrochloride in situ gel for uveitis treatment. Int. Immunopharmacol. 17, 99-107 https:/doi.org/10.1016/j.intimp.2013.05.020

Sowers J. R., Epstein M., Frohlich E. D. (2001): Diabetes, hypertension, and cardiovascular disease: an update. Hypertension 37, 1053-1059 https:/doi.org/10.1161/01.HYP.37.4.1053

Tong B., Yu J., Wang T., Dou Y., Wu X., Kong L., Dai Y., Xia Y. (2015): Sinomenine suppresses collagen-induced arthritis by reciprocal modulation of regulatory $\mathrm{T}$ cells and Th17 cells in gut-associated lymphoid tissues. Mol. Immunol. 65, 94-103 https:/doi.org/10.1016/j.molimm.2015.01.014

Wang Y., Fang Y., Huang W., Zhou X., Wang M., Zhong B., Peng D. (2005): Effect of sinomenine on cytokine expression of macrophages and synoviocytes in adjuvant arthritis rats. J. Ethnopharmacol. 98, 37-43 https:/doi.org/10.1016/j.jep.2004.12.022

Wang A. L., Li Z., Yuan M., Yu A.C., Zhu X., Tso M. O. (2007): Sinomenine inhibits activation of rat retinal microglia induced by advanced glycation end products. Int. Immunopharmacol. 7, 1552-1558 https:/doi.org/10.1016/j.intimp.2007.07.030

Wang Q., Li X. K. (2011): Immunosuppressive and anti-inflammatory activities of sinomenine. Int. Immunopharmacol. 11, 373-376 https:/doi.org/10.1016/j.intimp.2010.11.018

Wen H. L., Liang Z. S., Zhang R., Yang K. (2013): Anti-inflammatory effects of triptolide improve left ventricular function in a rat model of diabetic cardiomyopathy. Cardiovasc. Diabetol. 12, 50 
https:/doi.org/10.1186/1475-2840-12-50

Xu Z., Lin S., Wu W., Tan H., Wang Z., Cheng C., Lu L., Zhang X. (2008): Ghrelin prevents doxorubicin-induced cardiotoxicity through TNF-alpha/NF-kappaB pathways and mitochondrial protective mechanisms. Toxicology 247, 133-138 https:/doi.org/10.1016/j.tox.2008.02.018

Yang M., Chen J., Zhao J., Meng M. (2014): Etanercept attenuates myocardial ischemia/reperfusion injury by decreasing inflammation and oxidative stress. PLoS One 9, e108024 https:/doi.org/10.1371/journal.pone.0108024

Yu X. Y., Chen H. M., Liang J. L., Lin Q. X., Tan H. H., Fu Y. H. Liu X. Y., Shan Z. X., Li X. H., Yang H. Z., Yang M., Li Y., Lin S. G. (2011): Hyperglycemic myocardial damage is mediated by proinflammatory cytokine: macrophage migration inhibitory factor. PLoS One 6, e16239 https:/doi.org/10.1371/journal.pone.0016239

Yu W., Wu J., Cai F., Xiang J., Zha W., Fan D., Guo S., Ming Z., Liu C. (2012): Curcumin alleviates diabetic cardiomyopathy in experimental diabetic rats. PLoS One 7, e52013 https:/doi.org/10.1371/journal.pone.0052013

Yu L., Zhao Y., Xu S., Jin C., Wang M., Fu G. (2014): Leptin confers protection against TNF- $\alpha$-induced apoptosis in rat cardiomyocytes. Biochem. Biophys. Res. Commun. 455, 126 -132 https:/doi.org/10.1016/j.bbrc.2014.10.134

Zannad F., Briancon S., Juilliere Y., Mertes P. M., Villemot J. P., Alla F., Virion J. M. (1999): Incidence, clinical and etiologic features, and outcomes of advanced chronic heart failure: the EPICAL Study. Epidémiologie de l'Insuffisance Cardiaque Avancée en Lorraine. J. Am. Coll. Cardiol. 33, 734-742 https:/doi.org/10.1016/S0735-1097(98)00634-2

ZhangY., Sowers J. R., Ren J. (2012): Pathophysiological insights into cardiovascular health in metabolic syndrome. Exp. Diabetes. Res. 2012, 320534 https:/doi.org/10.1155/2012/320534

Zhao Y., Li J., Yu K., Liu Y., Chen X. (2007): Sinomenine inhibits maturation of monocyte-derived dendritic cells through blocking activation of NF-kappa B. Int. Immunopharmacol. 7, 637-645 https:/doi.org/10.1016/j.intimp.2007.01.007

Zhao X. X., Peng C., Zhang H., Qin L. P. (2012): Sinomenium acutum: a review of chemistry, pharmacology, pharmacokinetics, and clinical use. Pharm. Biol. 50, 1053-1061 https:/doi.org/10.3109/13880209.2012.656847

Zhao Z., Guan R., Song S., Zhang M., Liu F., Guo M., Yu Q., Zhang L., Wang Q. (2013): Sinomenine protects mice against ischemia reperfusion induced renal injury by attenuating inflammatory response and tubular cell apoptosis. Int. J. Clin. Exp. Pathol. 6, 1702-1712

Received: April 12, 2016

Final version accepted: July 15, 2016

First published online: November 30, 2016 